\title{
Kernos
}

Revue internationale et pluridisciplinaire de religion grecque antique

$2 \mid 1989$

Varia

\section{Jeux de mots et sens du sacré dans la religion grecque}

\section{Pierre Somville}

\section{(2) OpenEdition \\ Journals}

\section{Édition électronique}

URL : http://journals.openedition.org/kernos/249

DOI : $10.4000 /$ kernos. 249

ISSN : 2034-7871

\section{Éditeur}

Centre international d'étude de la religion grecque antique

\section{Édition imprimée}

Date de publication : 1 janvier 1989

Pagination : 199-211

ISSN : 0776-3824

\section{Référence électronique}

Pierre Somville, "Jeux de mots et sens du sacré dans la religion grecque », Kernos [En ligne], 2 | 1989, mis en ligne le 02 mars 2011, consulté le 01 mai 2019. URL : http://journals.openedition.org/ kernos/249; DOI : 10.4000/kernos.249 
Kernos, 2 (1989), p. 199-211.

\title{
JEUX DE MOTS ET SENS DU SACRÉ \\ DANS LA RELIGION GRECQUE
}

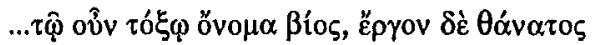 \\ ... ainsi l'arc porte nom de "vie", mais son \\ ceuvre est la mort . \\ HéRACLITE, D.K., 22 B 48.
}

Aucune fable ne m'a jamais paru plus significative de l'esprit grec que ce méchant tour joué par Ulysse au Cyclope lorsqu'il lui dit se nommer Personne. L'autre, aveuglé, appelle ses frères à la rescousse en disant que "Personne le malmène», mais ils rient - comme de vrais Grecs - évitant avec astuce de tomber dans le piège linguistique. En tout cas, c'est le jeu du langage et la référence oblique au réel qui permettent que Personne soit quelqu'un et que ce quelqu'un, grâce à son maquillage verbal, se sauve et sauve ses compagnons. L'heureux paradoxe, concerté et contrôlé, débouche donc sur une action parfaitement bénéfique.

Tel est aussi, je crois, le sens du "sourire archaïque». Considérons la Dame d'Auxerre et l'Apollon de Ténéa. Comment ne pas penser que cette aisance juvénile, à peine conquise sur un monde désormais ami, ne passe d'abord par la maîtrise du langage, d'un langage dissocié des faits et des gestes ? C'est le recul que prend l'intelligence vis-à-vis des choses qui peut, seul, permettre cette forme de confiance. Sinon, dans la "lutte avec l'ange", comment garder le sourire en affrontant les dieux, la mort, le destin ?

Cette aisance dans la maîtrise du langage, confinant parfois à la jonglerie pure et simple, semble traverser toute la culture grecque, puisque nous la retrouverons, à son terme, chez Denys d'Halicarnasse ou Plutarque qui ne manquent pas de relever à propos du vocable 'Pó $\mu \eta$ l'homonymie fortuite de la Ville et de la force. Or, pareille attitude aura souvent servi à maîtriser des forces inconnues, à focaliser en un même point d'émergence du sacré des pulsions diverses et parfois convergentes. Tantôt le processus est conscient, comme dans le cas d'Ulysse ou de Plutarque (dans la Vie de Romulus), tantôt le travail linguistique est souterrain ou sous-jacent et n'affleure qu'en quelques endroits précis où le temps, le lieu et la désignation d'êtres disparates se rencontrent sous un même vocable.

L'auteur de l'Hymne à Apollon, lui-même, nous indique cette herméneutique lorsqu'il propose au toponyme de Pytho et à l'épiclèse Puthios l'étymologie renvoyant au pourrissement $(\pi v \dot{\theta} \theta)$ du monstre Python tué par 
le dieu' ${ }^{1}$ S'il ne fait nul doute qu'une telle étymologie soit naïve, et donc "populaire», dans sa référence au langage, il n'en reste pas moins qu'elle témoigne d'une sensibilité chthonienne des plus justes. Le cycle archaïque de la vie et de la mort s'y trouve célébré : c'est du monstre abattu et de sa décomposition que surgit à nouveau la vie, sous les espèces du dieu de lumière purifié de son meurtre, ou plutôt, régénéré par sa mixis avec la terre noire et maternelle. Il supplante le «serpent» qui n'en était que l'avatar ou l'indigne "parèdre». Nous découvrons ainsi deux niveaux de lecture différents d'un ătน ov linguistique, l'un banal et concerté, l'autre à-demi caché et plus riche qu'il n'y paraissait au premier abord.

En deux autres exemples, conscients et reconnus, Penthée sera dès le texte

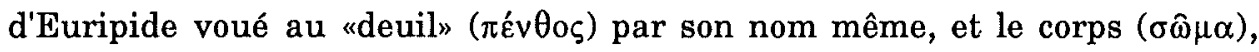
chez les Orphiques et Pythagoriciens dont Platon se fera l'écho, sera nommément conçu comme le tombeau ( $\sigma \hat{\mu} \mu \alpha)$ de l'âme ${ }^{2}$.

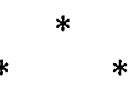

Examinons à présent quelques cas moins connus d'homonymies, mais non moins significatifs, dont les Grecs eux-mêmes ne semblent pas avoir été conscients. L'un ou l'autre point d'histoire des religions y gagnera, espérons-le, quelque éclairage. C'est bien d'homonymie, et mieux encore d'homophonie, qu'il s'agira, puisque nous verrons se nouer sous la même forme linguistique des réalités diverses. C'est ainsi que pratique, par jeu, toute espèce de calembour ou de rébus, mais dans les cas qui vont nous occuper, si la gratuité ludique semble parfois présente, elle s'effacera bien vite devant le signifié - unique et divin - qui lui servira de chiffre.

1 Voir dans la Suite Pythique les vers 372-374.

2 Voir dans les Bacchantes les vers 507-508. Pour l'allusion au corps «tombeau» de l'âme, voir Platon, Cratyle, 400c et Gorgias, 493a. De plus, cette image sous-tend tout le Phédon. Pour une bonne approche du sujet et quelques références supplémentaires, relatives à la tragédie (Esch., Prom, 85-86; SoPH., Aj., 430-431; EUR., Trag., 989-990 notamment), on consultera l'éclairant travail de M. WARBURG, Zwei Fragen zum "Kratylos», in Neue philologische Untersuchungen, fünfter Heft, Berlin, 1929 , surtout en sa seconde partie «Voraussetzungen zum Verständnis der griechischen Etymologie» (p. 65-121). L'auteur y est particulièrement attentif au contenu philosophique et religieux de ce qu'il appelle si justement «la mythologie des noms" (p. 102). Quant aux incessants jeux de mots d'Aristophane, de même que nos jurons ne sont souvent que des formules de serments dégénérées, nous les considérons sans plus comme la dévalorisation même du sujet qui nous occupe. Ajoutons que M. WALBURG ne les mentionne même pas. 
Pensons aux attributs d'Athéna et à la relative difficulté que pose l'interprétation de son épithète $\gamma \lambda \alpha v \kappa \hat{\omega} \pi \iota 5$. A-t-elle une face de chouette ou simplement les yeux verts (ou "pers" comme le dit si joliment Bérard) ? Si nous interrogeons vers l'amont cette figure divine, nous lui trouvons, pardelà cette Walkyrie indo-européenne qui "brandit" les armes (Pallas), une possible origine méditérranéenne et plus précisément crétoise, sous la forme d'une déesse de l'oiseau et de l'arbre. Lointain dérivé de cette Пó $\tau \imath \iota \alpha$ des origines, l'Athéna classique garde effectivement parmi ses rôles principaux celui d'avatar d'une Grande-Déesse ${ }^{3}$. Dès lors, qu'elle ait dû, un moment donné, être associée à un oiseau et à un arbre ne semble pas faire de doute. Or, si cet oiseau fut la chouette et cet arbre l'olivier, la chose est peut-être due au fait qu'en Grèce ces deux réalités portent le même nom ou qu'à tout le moins deux mots parfaitement identiques puissent les évoquer. Le mot qui désigne la chouette ( $\gamma \lambda \alpha v \kappa o ́ \varsigma)$, employé au génitif, - qui peut être considéré comme d'appartenance ou d'attribution, ou encore comme la matrice des autres cas de la flexion, - se trouve être le parfait homonyme de l'adjectif $\gamma \lambda \alpha v \kappa o ́ \varsigma$ (également oxyton) qui désigne la couleur verte de l'olive et des feuilles de l'olivier. Comment ne pas penser que, saisis par cette homophonie comme par une merveille de nature, les Grecs, soucieux d'associer un oiseau à un arbre, aient précisément choisi ces signifiants homophones pour exprimer la double relation sacrée de la Пó $v \imath \_\alpha$ aux règnes végétal et animal ? Dès lors, l'épiclèse $\gamma \lambda \alpha v \kappa \hat{\pi} \pi \iota \varsigma$ revêtirait, elle aussi, le double sens : la verdeur et l'oiseau nocturne pourraient s'y lire conjointement, comme en filigrane.

Une autre assimilation, d'une telle lisibilité et d'une telle évidence qu'on s'étonne à la voir uniment passée sous silence, concerne la naissance d'Apollon dans l'île de Délos. On se souvient du début de l'Hymne et de la longue énumération des lieux qui se refusaient à accueillir le dieu. C'est Délos, enfin, qui accepte, comme la dernière et la plus modeste des îles, celle à laquelle on n'aurait pas pensé. Or, nous assistons à un travail mythique d'occultation assez extraordinaire, puisqu'en fait, par son nom même, l'île devait, par prédestination, être prêtée et vouée au dieu de lumière. Étymologiquement, $\Delta \hat{\eta} \lambda$ os est le nom d'un lieu placé sous le reflet divin, dans le

3 Voir M.P. NILsson, Minoan-Mycenaean Religion and its survival in the Greek religion, Lund, 1949, 2e éd., p. 277 sq., 330 sq., 489 sq. 
rayon lumineux que confère l'évidence dorée des dieux de l'Olympe et des autres déesses! Les longues et infructueuses pérégrinations de Latone ne font que mettre davantage en évidence cette rencontre homophonique du lieu, luimême sec et lumineux, et du futur dieu solaire qui en sera l'hôte. $\Delta \hat{\eta} \lambda \chi_{o \zeta}$ et Фoîßoc sont d'ailleurs presque synonymes et d'autant plus complémentaires qu'ils diffèrent par le genre : Délos est féminine, comme toutes les îles, et Phœbos, masculin. De leur conjonction, réunissant les deux moitiés jumelles d'un englobant lumineux, surgit dans sa perfection le sumbolon aux deux moitiés appariées. Le texte même de l'Hymne suggère ce rapprochement lorsque Latone, dans sa prière à Délos, profêre en tête de vers et au vocatif le nom de l'île, puis en tête du vers suivant, énonce, au génitif, le nom de Phobos comme si chacun de ces termes était le redoublement ou la redondance de l'autre :

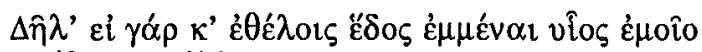

Фoíßov 'A $\pi$ ó $\lambda \lambda \omega v o s . .$.

(vv. 51-52)

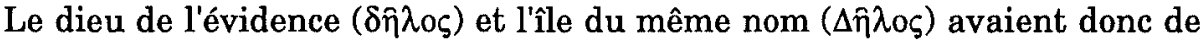
secrètes accointances lexicales, capables de rendre compte d'un aspect, au moins, de l'aîtนtov du mythe.

Après l'hymne délien, la Suite pythique que nous réserve un bel exemple de la même démarche onomastique. J'en ai parlé ailleurs ${ }^{4}$. Il s'agit de la double relation du dieu Apollon au dauphin et à Delphes. Or, le rapport linguistique possible entre le toponyme, le nom de l'animal et celui de la matrice ${ }^{5}$ est un lieu commun de l'étymologie savante. Mais ce qui n'avait jamais été dit, je crois, est la stricte homonymie des deux acceptions du vocable $\delta \varepsilon \lambda \varphi$ í signifiant soit la terre de Delphes soit le dauphin. Et nous mettons le doigt, à nouveau, sur cette forme de calembour sacré (ou sacralisateur). De même ici, sous l'apparente banalité d'un simple jeu de mots, nous pourrons lire, en plus du $\theta \alpha \alpha \beta \beta$ ó que devait suggérer ce phénomène

4 Voir P. SOMVILLE, Le dauphin dans la religion grecque, in RHR, 201 (1984), p.3-24.

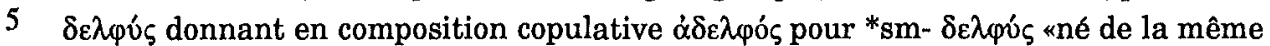
matrice». Voir E. BoISACQ, Dictionnaire étymologique de la langue grecque, Paris, 1916, p. 174-175. 
lexical fortuit et quasi divinatoire, une grande richesse de contenu sémantique. La terre (au féminin) y est associée au merveilleux mammifère marin (masculin), comme si, à nouveau, les deux moitiés jumelles d'un même imaginaire archaïque cherchaient à reformer leur totalité perdue. Le dieu crétois, delphinomorphe et parèdre de la déesse, devient ainsi tout naturellement l'ultime occupant de la Terre de Delphes et de son oracle.

L'arbre même au pied duquel accouchait la déesse, en l'île de Délos, nous réserve, lui aussi, une belle moisson polysémique. Mais relisons d'abord ces quelques superbes vers de l'Hymne à Apollon:

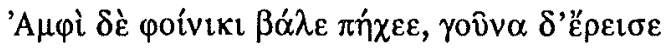

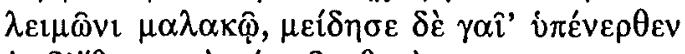

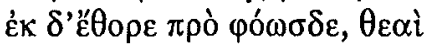

$\delta$ 'ỏ $\lambda \hat{o ́} \lambda v \xi \alpha \nu ~ \alpha ँ \pi \alpha \sigma \alpha$.

Embrassant des deux bras le palmier, ses genoux

S'enfonçaient sous l'herbe tendre et la terre sourit par dessous

Tandis qu'à la lumière elle expulsait l'enfant

Et que criaient de joie ensemble les déesses...

(vv. 117-119)

On sait le rôle d'arbre de fécondité que joua le palmier-dattier dans tout le Proche-Orient ancien. On se souvient aussi de la comparaison homérique où Ulysse rapproche la noblesse et la grâce de Nausicaa (VI, 162-163) de celle d'un surgeon de palmier. Ce qu'on oublie plus souvent de dire, c'est l'écheveau foisonnant de significations qui se noue autour du terme poîvi $\xi$. L'écarlate, la pourpre et le sang n'y trouvent pas moins place que le dattier et l'oiseau fabuleux qui renaît de ses cendres ! L'origine orientale et "phénicienne» de toutes ces désignations explique peut-être la tonalité rouge de la plupart d'entre elles, puisqu'elles évoquent l'aurore et le levant, mais on est loin du compte si l'on s'en tient à ce genre d'accident de surface. En effet, ce sont des pans entiers de constructions mythiques que nous voyons s'élever autour de l'arbre et de la déesse, de l'écarlate et de la pourpre (qui célèbrent la vie), du sang répandu (qui signifie le meurtre ou le malheur), de l'oiseau de feu et d'aromates (qui se consume et retrouve la vie dans sa crémation même $)^{6}$. S'engendrant de lui seul, il est simultanément le fils et

6 Les principaux récits du mythe se trouvent en HDT, II, 73 et TACITE, Ann., VI, 28. Voir aussi le bel ouvrage de J. HubAux et M. Leroy, Le mythe du Phénix (dans les 
le père, en une seule personne... Pareille rencontre donne le vertige et bien qu'on sache toujours parfaitement si l'on parle du sang, de l'arbre ou de l'oiseau, on ne peut s'empêcher de percevoir, par-delà la dominante et la tonique, les diverses harmoniques qui ne manquent pas de résonner autour du thème.

Ainsi, pour faire bref et sortir un instant de la tradition héllénique, rappelons que l'arbre (palmier-dattier) entre en collusion avec l'oiseauphénix tout au long du Moyen Âge pour évoquer le Christ, sous le double aspect de la fécondité spirituelle et de la réalité de Fils-du-Père, ressuscité d'entre les morts : l'amalgame étant ainsi établi, c'est toujours ce double sens qu'il faut lire, dans l'iconographie gothique et pré-renaissante, lorsqu'on rencontre un palmier-dattier dans un de ces merveilleux paysages qui servent de fond aux épiphanies des "Primitifs». Tel est le sens du palmier sur l'un des volets du polyptyque des Van Eyck représentant le groupe des Pèlerins. Tel en est aussi le sens sur le panneau central du même Agneau mystique derrière le groupe des Confesseurs, juste un peu à gauche de la Jérusalem céleste. Sur un autre polyptyque, retable de Mathias Grünewald, la rencontre, soi-disant au désert, de saint Paul Ermite et de saint Antoine se passe en un décor de paysage parfaitement alpin, sauf ce détail : derrière le saint Ermite se dresse l'arbre de la fécondité spirituelle et du renouveau, emblème du Christ et de ses disciples.

Le détour par le Moyen Âge et la Renaissance nous ramène de droit à l'Antiquité et particulièrement à l'Antiquité latine, où l'homonymie existe également : le mot phoenix désigne aussi bien l'oiseau que le palmier. Toutefois, il s'agit de décalques du grec, dont le recouvrement se fera, avec le temps, de moins en moins perceptible. Le bilinguisme n'étant pas l'affaire des médiévaux (graecum, non legitur...) on ne s'étonnera pas du peu de représentations figurées de l'oiseau, par rapport à l'arbre notamment. Il n'en reste pas moins que la collusion symbolique - et non seulement lexicale - apparaît d'une manière assez inattendue, dès Ovide, ce maître à penser les images et les mythes, qui sera tant lu au Moyen Age et à la Renaissance :

Una est, quae reparet seque ipsa reseminet, ales :

Assyri "phønica» vocant; (...)

Haec ubi quinque suae complerit saecula vitae,

Ilicis in ramis tremulaeve cacumina palmae

Unguibus et puro nidum sibi construit ore.

littératures grecque et latine), Univ. de Liège et Paris, Droz, 1939. A propos de l'жarbre», voir H. DANTHINE, Le palmier-dattier et les arbres sacrés dans l'iconographie de l'Asie occidentale ancienne, Paris, Geuthner, 1937. 
Il en est un, qui naît de sa propre semence, Un oiseau, le "phénix», disent les Assyriens; (...)

Qui, lorsqu'il a vécu ses cinq siècles de vie,

Sur les branches d'une yeuse ou le sommet tremblant

D'un palmier,

Des ongles et du bec dûment purifié,

Va se construire un gîte...

(Métamorphoses, XV, 392-397)

On l'aura remarqué, les deux mots employés par le poète sont différents : bien que phœenix soit attesté pour désigner le palmier, c'est ici la forme métonymique palma qui se présente en parallèle avec l'ilex. Il ne peut en fait s'agir que de notre dattier : le détail visuel de sa "cime tremblante" suffirait à nous en évoquer l'indubitable silhouette. Ainsi l'association des deux poívıкes, pour traditionnelle qu'elle pût paraître, était déjà comme subconsciente chez le poète des Métamorphoses. Sans doute fut-il l'un des relais par lesquels l'amalgame symbolique se sera transmis aux siècles postérieurs. Guy de Tervarent semble cependant l'ignorer, dans son Dictionnaire, puisque les articles «Palmier» et «Phénix» ne suggèrent pas la moindre relation mutuelle ${ }^{7}$. Pourtant, en plus d'Ovide, Pline l'Ancien et Tacite, au moins, en attestaient l'homonymie latine ${ }^{8}$.

Voyons encore trois exemples de collusions lexicales qui, sans référence directe au panthéon classique, n'en ressortissent pas moins à la sphère du sacré. Il s'agit d'abord d'une coutume rituelle imputée aux Pythagoriciens. Hérodote nous apprend (II, 81), sous une forme un peu confuse, que les Égyptiens, les sectateurs d'Orphée et de Dionysos, et les Pythagoriciens évitaient soigneusement de porter des vêtements «de laine» pour entrer dans leurs temples ou se faire ensevelir. La chose est interdite pour raison

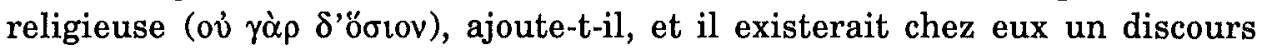
sacré (įpòs $\lambda o ́ \gamma o \varsigma)$ sur cette question.

7 G. de Tervarent, Attributs et symboles dans l'art profane (1450-1600), Genève, Droz, 1952, s.v. «Palmier» et «Phénix», resp. t. II, col. 295-297 et 304-306.

8 Voir Pline (H.N., XXIX, 56) pour l'arbre; TACITE (Ann., VI, 28) ainsi que SÉNEQUE (Ep., 42, 1) pour l'oiseau. 
Inutile de dire qu'on n'a retrouvé aucune trace de ce ípòs $\lambda$ ófos et que ce tabou a toujours paru, aux commentateurs, aussi incongru qu'inexplicable ${ }^{9}$. Il est vrai que les $\sigma v ́ \mu \beta 0 \lambda \alpha$ pythagoriciens obligent parfois à quelque détour herméneutique... Ici de même, que proposer, faute de mieux, qu'un calembour implicite ? Il pourra, peut-être, rendre compte de la sacralité prêtée à ces fameux vêtements. C'est que le mot signifiant «de laine» (Épíveos, en attique) est l'homonyme du terme signifiant la figuier ou la figue sauvage (épıveós, chez Homère notamment). Dès lors, on peut imaginer, par collusion sémantique, que la tonalité attachée à la figue et au figuier, - qui sont des attributs symboliques bien connus de la Grande-Mère des origines ${ }^{10}$, - ait pu affecter par contamination, un terme homophone avec lequel elle n'avait, au départ, absolument rien à voir. C'est une hypothèse et elle est fragile, je le

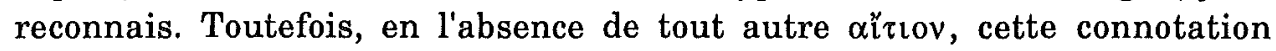
linguistique et le mince faisceau lumineux qu'elle représente balisent tant bien que mal une grande zone d'ombre. De plus, la démarche s'inscrit dans la continuité d'une série dont d'autres pièces, mieux établies, garantissent le bien-fondé.

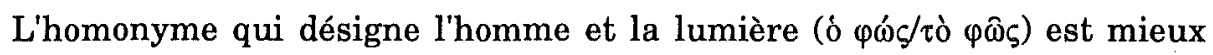
connu. Malgré le changement de genre et d'accent, la matrice du génitif ( $\varphi \omega \tau$ ós) est identique pour les deux termes et le sanskrit bhah signifiant à la fois la "lumière» et le «regard» plaide pour une racine commune donnant en grec $\varphi \alpha ́$ os, et dont dérivent nos deux termes. En effet, la sémantique, même sans recourir au sanskrit, nous apprend que le grec, - non content de considérer le symbolisme lumineux comme constitutif de la vie et comme emblème de l'homme debout sous le soleil, - utilise aussi le terme de $\varphi \hat{\omega} \tau \alpha$ pour désigner métonymiquement les yeux et le regard, et donc, métaphoriquement aussi, l'être humain. Je ne rappellerai pas ici toute cette symbolique de la lumière, constitutive de l'anthropologie hellénique, d'Homère à Platon et d'Aristote à Plotin, mais je citerai seulement un passage de Parménide, riche de telles connotations :

9 Voir notamment P. Boyancé, Le culte des Muses chez les philosophes grecs, Paris, 1937, p. 94. L'auteur y suggère aussi qu'Hérodote transpose à l'Égypte cette coutume d'origine grecque...

10 Voir, en plus du fr. 11 d'Archiloque (Lasserre), U. Pestalozza, Religione mediterranea, Milan, 1971 (rééd.), p. 368 sq. notamment. Comme le rappelle également A. Mотте, Prairies et Jardins de la Grece antique, Bruxelles, 1973, p. 239, n. 17 : «Dans la plaine d'Éleusis, on appelait Erinéon (figuier) l'endroit où la terre s'était ouverte pour livrer passage à Hadès» (PAUS., I, 38, 5). 


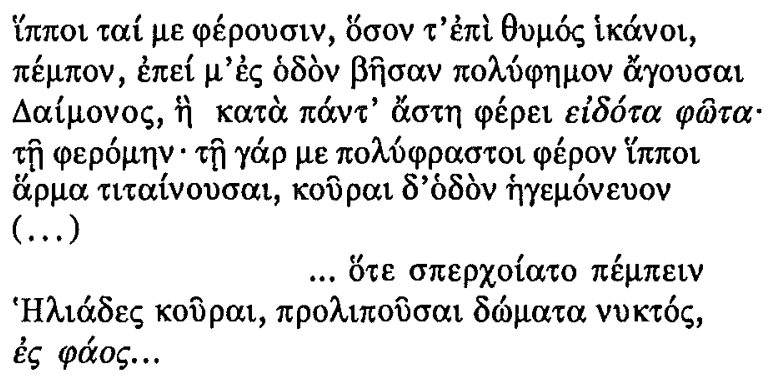

Les cavales qui m'emportent, aux fins de mon vouloir

M'avaient conduit, car elles allaient m'entraînant sur la voie entre toutes fameuse

De la Déesse, qui par tous lieux habités porte l'homme qui sait

Ainsi étais-je emmené, ainsi m'emportaient ces parlantes cavales, Elles tiraient le char, des vierges indiquaient la voie

(...)

Ainsi à chaque fois que m'emmenaient en toute hâte

Les filles du Soleil, qui avaient laissé là les demeures de Nuit,

Pour la lumière...

(D.K., 28 B 1, 1-5; 8-10)

Le merveilleux prologue du poème s'ouvre ainsi sur l'évocation d'un voyage

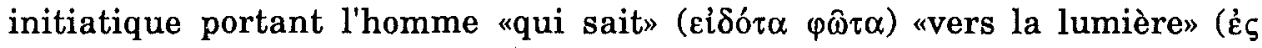

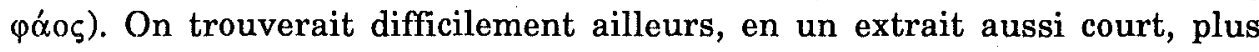
riche moisson de connotations lumineuses. Si l'on se rappelle que la racine commune de oî $\delta \alpha$ et de uidere évoque directement la vision, l'homme-voyant destiné à l'initiation, lumineuse elle aussi, ne fait que redire cette aspiration "visuelle" au savoir eidétique et contemplatif. Toute la Grèce est là in nuce : celle du mythe de la Caverne, comme celle, déjà byzantine, des esthétiques de la lumière...

Reste l'exemple du papillon, curieux à plus d'un titre. Le nom de l'âme, également associé au vocable $\psi v \chi \eta ́$, semble en effet avoir entièrement supplanté l'autre signification de papillon, ou plus précisément, de phalène ${ }^{11}$. A tel point que certains textes de sciences naturelles qui nous

11 L'homonymie n'y est en aucun cas évoquée ni même effleurée. Platon pense à tout autre chose dans le Cratyle (399d). Pour l'expression koccultée», voir ARIST., $H$. $A$,

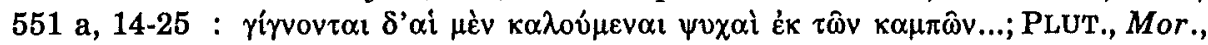

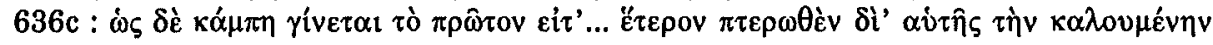


parlent de larves et de chrysalides ne peuvent s'empêcher, lorsqu'ils en viennent à la métamorphose ultime, de recourir à la périphrase $\alpha i$ $\kappa \alpha \lambda \circ u ́ \mu \varepsilon v \alpha \iota$ $\psi v \chi \alpha$ í, comme s'ils voulaient par là s'excuser d'une homonymie indécente et inacceptable. Étrange occultation, à la vérité, qui fait presque penser à un phénomène de refoulement : il est vrai que l'image post mortem d'une phalène, vouée à se faire dévorer par la flamme, a de quoi inquiéter une âme archaïque. Toujours est-il que ce double sens, pourtant évident, n'est attesté nulle part à ma connaissance. Au contraire, c'est la précaution périphrastique qui est de règle et semble fonctionner comme un véritable exorcisme. Pourtant, la réalité naturelle, qui préexiste au langage, est bien évidente : elle montre le changement d'état et l'envol à partir d'une apparente décomposition. Quoi qu'il en soit des étymologies, il semble clair que l'observation de ce phénomène naturel ait dû provoquer très tôt l'association analogique avec le cadavre et cette fraîcheur du dernier souffle qui s'envole des lèvres du mourant. Le mot est là pour nous le rappeler, même si les Grecs de l'époque historique semblent avoir voulu en refuser la vision. «Le soleil ni la mort ne se peuvent regarder fixement», dira La Rochefoucauld...

En amont, toutefois, l'archéologie mycénienne nous révèle quelques beaux exemples de feuilles d'or battu décorées de papillons et retrouvées dans les tombes, indice de leur probable destination funéraire ${ }^{12}$. En aval, chez les Latins, on trouve de nouveau des exemples d'assimilation analogique de l'âme au papillon. Mais là, le tabou de l'homonymie a disparu, et c'est de papilio que l'on nous parle. Le sens de métaphore funèbre dont Ovide en colore l'évocation porte tout son poids :

Quaeque solent canis frontes intexere filis

Agrestes tineae (res observata colonis)

Ferali mutant cum papilione figuram...

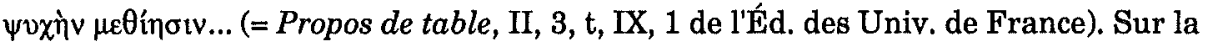
signification de "phalène», voir le Thesaurus graecae linguae (Didot, 1865) t. VIII, s.v.

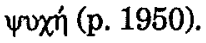

12 Voir au Musée d'Athènes la pièce 5619 (vitrine 48) provenant d'une tombe thessalienne et diverses pièces d'or battu provenant de la tombe III de Mycènes (vitrine 23). Cf. le Catalogue d'A. SAKELlariou (Collections préhistoriques, Athènes, 1965, p. 34-35). Dans une autre aire culturelle, celle de la méso-Amérique, le thème funéraire du papillon est très bien représenté, dès Teotihuacan et jusque chez les

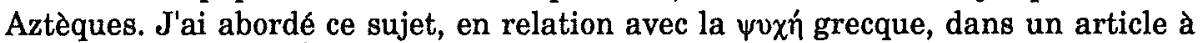
paraître dans Les Études Classiques (Namur) écrit en collaboration avec J.-C. DELHALLE et A. LUYCX : Croyances grecques et mexicaines. 
Et les chenilles des champs tissant autour des feuilles Les fils blancs (chose commune à la campagne)

On les voit se changer en papillon de mort...

(Métamorphoses, XV, 372-374)

Toute la force du témoignage réside dans l'adjectif ferali qui est apparemment gratuit et scientifiquement inexact, mais symboliquement probant, puisqu'il fait porter à l'image de l'âme libérée la contamination du cadavre dont elle est issue.

De même, une fable de Phèdre consacrée aux métamorphoses post mortem d'une guêpe et d'un papillon fait dire à ce dernier, regrettant le corps vivant dont il fut expulsé :

En cassa levitas putris et volito cinis.

Hélas je ne suis plus qu'une chose légère,

Creuse et décomposée, et je volète de ci-de là,

Comme une cendre.

(Fable 134)

L'allusion funéraire à la cendre est également très forte, et tout se passe comme si la dissociation verbale entre l'âme et le lépidoptère, impossible en grec mais naturelle en latin, libérait l'expression de cette réalité douloureuse, et désormais possible à dire.

L'un ou l'autre texte d'inscription funéraire nous en fournirait également le témoignage ${ }^{13}$. Enfin, un exemple grec, contemporain ou presque, nous permettra de renouer le fil hellénique à notre écheveau. C'est un document iconographique que je veux produire ici et qui nous fera ressou-

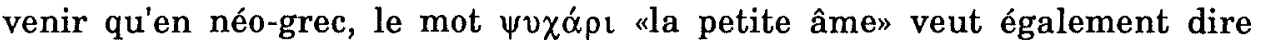
«papillon»... Il s'agit du monument funéraire de Lazaros Kondouriotis, situé dans la cour du monastère d'Hydra, qui célèbre le défenseur de l'île contre les Turcs (en 1821). Or, les deux frontons de ce sarcophage sont ornés de papillons en leur centre. On y trouve associées, sur les flancs, d'autres images de vie éternelle et de pureté : le lychnos, l'amphore, le chrisme et la rosace. Ce décor, dédié en plein XIXe siècle à un héros local (mort en 1852), plaide en tout cas pour une belle continuité symbolique et nous montre, contrairement à ce que parfois l'on pense, que les images font moins peur que les mots.

13 C.I.L., II, 2146 ut...volitet meus ebrius papilio (après une libation de vin). 
Enfin, on s'étonnera peut-être de n'avoir pas encore trouvé mention du Cratyle. Voici. Sur fond de problème philosophique posant, déjà, le dilemme du réalisme et du nominalisme, Platon nous y offre, un peu comme le Varron du De lingua latina, de gratuites et étourdissantes étymologies, fantaisistes à souhait. Par provocation "socratique», à n'en pas douter. Toutefois, au détour d'une de ces brillantes passes d'armes philosophico-linguistiques (où Socrate tient le plus souvent les deux rôles à lui tout seul), on voit pointer la peur $d u$ mot et ce $\theta \alpha \dot{\alpha} \mu \beta$ o̧ sacré dont une homonymie peut être porteuse. Il s'agit de l'étymologie du nom d'Apollon (en 405d-e) dont la connotation funeste se trouve attestée déjà chez Eschyle ${ }^{14}$. Or, entre autres hypothèses cratyléennes, Socrate propose celle-ci : puisqu'il est le dieu de l'harmonie (dans des domaines aussi différents que la musique, la médecine, le tir à l'arc et la mantique), quelque chose dans son nom même devrait bien le suggérer. Il propose alors de lire sous le nom du dieu «celui-qui-relie-les-pôles-opposés». Il mentionne à cet effet l'interprétation de l'alpha copulatif (comme dans

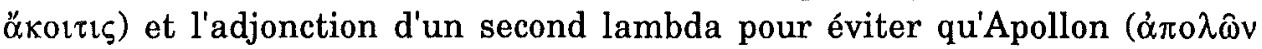
au participe futur) ne devienne "celui-qui-fait-périr». Et dès lors les rieurs se taisent, car - foin de la «linguistique amusante» - le dieu de Delphes est bien à la fois celui qui harmonise les contraires et fait périr, tantôt les mulets et les chiens, comme en Troade, et tantôt, à Thèbes, le pauvre et glorieux (Edipe. Ce passage est d'une grande force, car, parmi le divertissement quasi sophistique de tous ces jeux verbaux, Socrate (et Platon) mettent précisément le doigt sur la stupeur sacrée qui fait de toute âme religieuse une inconditionnelle adepte de Cratyle. De fait, les noms communs sont parfois de véritables noms propres, doués d'un statut de signatures. C'est du lexique que vient alors l'apothéose $1 \mathrm{Et}$ nous sommes renvoyés, nommément, à la mantique et aux oracles. C'est là la méthode d'Euthyphron et de ses amis, ironise-t-on par deux fois ${ }^{15}$. Pourtant, loin de toute bigoterie, il reste que le seul vrai domaine cratyléen est celui de l'archaïsme religieux. Le mot y est efficace, même s'il faut parfois s'en écarter, pour en mieux jouer, s'en protéger, le rendre bénéfique ou apotropaïque...

Vraiment, et comme après saint Jean le redira superbement Luther, sans équivoque possible :

\section{Im Anfang war das Wort!}

D'ailleurs, pour nous en tenir à la mantique dont Platon fait ailleurs ${ }^{16}$ jouer le reflet «démentiel» ( $\mu \alpha v i ́ \alpha / \mu \alpha \nu \tau \imath \kappa \eta ́)$, ajoutons que, si la fameuse

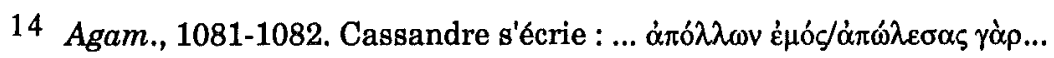

15 En 384a et 396d.

16 Phedre, 224b-c. 
Diotima du Banquet (201d) est dite "originaire de Mantinée», ce qui fait

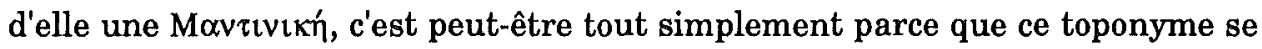

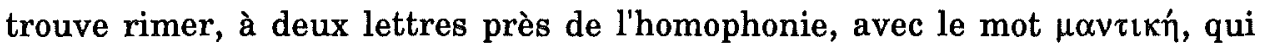
évoque la faculté oraculaire de cette devineresse qui va nous révéler l'amour...

Université de Liège

Pierre SOMVILLE

Place du XX-Août, 32

B - 4000 LLËGE 